H. Tamura

Nagoya Math. J.

Vol. 71 (1978), 125-147

\title{
ON THE DECAY OF THE LOCAL ENERGY FOR WAVE EQUATIONS WITH A MOVING OBSTACLE
}

\author{
HIDEO TAMURA
}

\section{§. Introduction}

Recently the decay of the local energy for wave equations with a moving obstacle $\mathcal{O}(t)$ has been studied by Cooper [1] and Cooper and Strauss [2] etc. In their works it has been assumed that the obstacle $\mathcal{O}(t)$ is uniformly bounded in time $t$ and that the origin is contained in $\mathcal{O}(t)$ for all $t>0$ and $\mathcal{O}(t)$ is star-shaped with respect to the origin. (The second condition has been assumed implicitly in [2] (see Assumption (B), [2]).)

The purpose of this paper is to give a slight extension of their works in the following two aspects: (i) We deal with a expanding obstacle with time (Assumption (4) stated in §1). (ii) We do not assume that the origin is contained in the obstacle for all $t$. Instead, we assume that there exists a point $a(t)$ satisfying Assumptions (2) and (3) in the obstacle for each $t$ (see $\S 1$ ). These assumptions are roughly stated as follows: The obstacle is star-shaped with respect to $a(t)$ and $a(t)$ moves slowly with time. However, we admit $a(t)$ to go to infinity as $t \rightarrow \infty$. The more precise assumptions on the obstacle $\mathcal{O}(t)$ are made in $\S 1$ and the main result is stated there.

\section{§1. Assumption and main result}

First we shall introduce some notations and make several assumptions on the moving obstacle.

Let $\mathcal{O}(t), t \geq 0$, be a bounded domain in $R^{3}$ with smooth boundary and let $\mathscr{E}(t)$ be a domain exterior to $\mathcal{O}(t)$. We denote by $\Sigma(t)$ the boundary of $\mathscr{E}(t)$. Let

$$
\mathscr{E}=\bigcup_{0<t<\infty} \mathscr{E}(t) \times\{t\}
$$

Received July 4, 1977. 
We denote by

$$
\Sigma=\bigcup_{0<t<\infty} \Sigma(t) \times\{t\}
$$

the lateral boundary of $\mathscr{E}$ and assume that $\Sigma$ is smooth. For each fixed $s \geq 0$, we introduce the notations $\mathscr{E}_{s}(0, T)$ and $\Sigma_{s}(0, T), 0<T \leq \infty$, as follows :

$$
\mathscr{E}_{s}(0, T)\left(\Sigma_{s}(0, T)\right)=\bigcup_{0<t<T} \mathscr{E}(s+t)(\Sigma(s+t)) \times\{s+t\} .
$$

In particular, when $T=\infty$, we write $\mathscr{E}_{s}=\mathscr{E}_{s}(0, \infty)$ and $\Sigma_{s}=\Sigma_{s}(0, \infty)$. In order to clarify the fixation of $s$, we occasionally write $\mathscr{E}(s+t)$ and $\Sigma(s+t), t \geq 0$, as $\mathscr{E}(t ; s)$ and $\Sigma(t ; s)$, respectively.

We denote by $n=\left(n_{1}, n_{2}, n_{3}, n_{t}\right)$ the exterior unit normal to $\mathscr{E}$ on $\Sigma$ and write $n_{x}=\left(n_{1}, n_{2}, n_{3}\right)$.

Assumption (1). $\quad \Sigma$ is time-like, that is $\left|n_{t}\right|<\left|n_{x}\right|$ for each $(x, t) \in \Sigma$, $\left|n_{x}\right|$ being the length of $n_{x}$.

Assumption (2). There exists a point $a(t)=\left(a_{1}(t), a_{2}(t), a_{3}(t)\right)$ in $\mathcal{O}(t)$ with the following properties:

$$
\left|a_{t}(t)\right|=\left(a_{1 t}(t)^{2}+a_{2 t}(t)^{2}+a_{3 t}(t)^{2}\right)^{1 / 2} \leq \mu, \quad \mu<1,
$$

for $t \geq 0$, where $a_{j t}(t)=\frac{d}{d t} a_{j}(t), \quad j=1,2,3$;

$$
\left|a_{j t}(t)\right| \leq C(t+1)^{-\beta}, \quad \text { and } \quad\left|a_{j t t}(t)\right| \leq C(t+1)^{-1-\beta}, \quad 0<\beta \leq 1 ;
$$

(1.3) $\mathcal{O}(t)$ is star-shaped with respect to $a(t)$.

We introduce the notation: For $x=\left(x_{1}, x_{2}, x_{3}\right)$

$$
r(t)=|x-a(t)| \text { and } z_{j}(x, t)=\frac{x_{j}-a_{j}(t)}{r(t)} .
$$

Then the condition (1.3) is stated as follows:

$$
n_{r(t)}=z_{j}(x, t) n_{j} \leq 0
$$

for $(x, t) \in \Sigma$, where we have used the summation convention.

Assumption (3). There exists a constant $\sigma_{0}, 0<\sigma_{0}<1$, such that for $(x, t) \in \Sigma$

$$
n_{t}+\sigma_{0} n_{r(t)} \leq 0
$$


If we assume that $\mathcal{O}(t)$ is uniformly (strongly) star-shaped in $t$ with respect to $a(t)$, that is $n_{r(t)} \leq-\sigma_{1}\left|n_{x}\right|, 0<\sigma_{1}<1$, then (1.5) follows from the condition

$$
n_{t} \leq \sigma_{2}\left|n_{x}\right|
$$

for some $\sigma_{2}, 0<\sigma_{2}<\sigma_{1}$. If $\mathcal{O}(t)$ is a ball with radius $\rho(t)$, then we can take $a(t)$ as the center of $\mathcal{O}(t)$ and $\sigma_{0}$ close enough to 1 .

Assumption (4). $\mathcal{O}(t)$ satisfies

$$
\left\{x \mid r(t) \leq \gamma_{0}\right\} \subset \mathcal{O}(t) \subset\left\{x \mid r(t) \leq(t+\gamma)^{\alpha}\right\}
$$

for each $t \geq 0$, where $0 \leq \alpha<1, \gamma>1$ and $0<\gamma_{0}<\gamma^{\alpha}$.

The constants $\alpha, \beta, \gamma, \gamma_{0}, \mu$ and $\sigma_{0}$ are used with the meanings ascribed here throughout this paper.

Now, under Assumptions (1) (4) stated above, we consider the following equation :

$$
\begin{gathered}
u_{t t}-\Delta u=0 \quad \text { in } \mathscr{E} \\
u=0 \quad \text { on } \Sigma \\
u(x, 0)=f(x), \quad u_{t}(x, 0)=g(x) \quad \text { on } \mathscr{E}(0) .
\end{gathered}
$$

Here the initial data $f$ and $g$ are assumed to be of compact support and to belong to $H_{0}^{1}(\mathscr{E}(0))$ and $L^{2}(\mathscr{E}(0))$, respectively. It is known that under this condition for initial data, the above problem has a unique (weak) solution such that for any fixed $T$

$$
u \in C\left([ 0 , T ] ; H _ { 0 } ^ { 1 } ( \mathscr { E } ( t ) ) \quad \text { and } \quad u _ { t } \in C \left([0, T] ; L^{2}(\mathscr{E}(t))\right.\right. \text {. }
$$

Furthermore, if the initial data $f$ and $g$ satisfy the compatible condition of infinite order, the solution $u$ is smooth. And also, a weak solution with the above property is obtained as a limit of such a smooth solution in the energy norm. ([1], [3])

Next, for fixed $s \geq 0$, we consider the following equation:

$$
v_{t t}-\Delta v=0 \quad \text { in } \mathscr{E}_{s},
$$

$$
v=0 \quad \text { on } \Sigma_{s} \text {, }
$$

$(\mathrm{P} .3 ; s) \quad v(x, 0 ; s)=f(x ; s), \quad v_{t}(x, 0 ; s)=g(x ; s) \quad$ on $\mathscr{E}(0 ; s)$,

where the initial data $f(x ; s)$ and $g(x ; s)$ are assumed to satisfy the 
same conditions as $f(x)$ and $g(x)$ in (P.3). We denote by $v(t ; s)$ a solution of problem $(\mathrm{P} .1 ; s) \sim(\mathrm{P} .3 ; s)$. For the solution $v(t ; s)$, we define the local energy measured over $B(h ; s)=\{x \mid x \in \mathscr{E}(T ; s), r(T ; s) \leq h\}$, $r(T ; s)=r(T+s)$, at $t=T$ as follows:

$$
E(v ; h, T, s)=\int_{B(h ; s)}\left(\left|v_{t}(T ; s)\right|^{2}+|\nabla v(T ; s)|^{2}\right) d x
$$

Let $h>0$ be fixed and let

$$
D(T ; h)=\left\{x \mid x \in \mathscr{E}(T), r(T) \leq(T+\gamma)^{\alpha}+h\right\},
$$

where $D(T ; h)$ is not void by (1.6). Then, the main result can be roughly stated as follows:

MAIN THEOREM. Under Assumptions (1) (4), the local energy measured over $D(T ; h)$ for solutions of problem (P.1) $\sim(\mathrm{P} .3)$ decays at the rate of $\exp \left(-M T^{\theta}\right), 0<\theta \leq 1$, as $T \rightarrow \infty$.

The explicit expression of the constant $\theta$ will be given in the proof of this theorem (Theorem 5).

The proof of Main Theorem is based on the "so-called" energy method. In $\S 2$ we prove several energy inequalities and from these inequalities we deduce that the local energy decays at the rate of $T^{-\nu}$, $\nu>0$, as $T \rightarrow \infty$. In $\S 3$, we prove Main Theorem in the way used by Morawetz [5] and modified by the author [6].

Finally we note the following facts throughout this paper: (a) The symbols $C, C_{1}, C_{2}, \cdots$ are used to denote (unessential) positive constants, which are not necessarily the same; (b) we use the summation convention; (c) All the functions considered here are real-valued.

\section{§2. Energy estimate}

First we recall the notations $r(t)$ and $z_{j}(x, t)$ introduced by (1.4) and set

$$
r(t ; s)=r(t+s) \quad \text { and } \quad z_{j}(x, t ; s)=z_{j}(x, t+s)
$$

for fixed $s \geq 0$. Furthermore we introduce the notation:

$$
u_{r(t ; s)}=z_{j}(x, t ; s) u_{j}
$$

where $u_{j}=\frac{\partial}{\partial x_{j}} u, j=1,2,3$. 
The next lemma is easily proved by (1.1) and (1.2).

LEMMA 2.1. (i) For $\mu, \mu<1$, introduced in (1.1)

$$
|a(t)-a(s)| \leq \mu|t-s| \text {. }
$$

(ii) There exists a constant $C$ for which the following estimates hold:

$$
\begin{gathered}
\left|r_{t}(t ; s)\right| \leq C(t+s+1)^{-\beta} \\
\left|r_{t t}(t ; s)\right| \leq C\left(r(t ; s)^{-1}(t+s+1)^{-\beta}+(t+s+1)^{-1-\beta}\right) \\
\left|z_{j t}(x, t ; s)\right| \leq C r(t ; s)^{-1}(t+s+1)^{-\beta}
\end{gathered}
$$

The following identity plays an important role in the proof of energy estimates.

Proposition 1 (cf. Zachmanoglou [7]). Let $s \geq 0$ be fixed and let $u(x, t)$ be a $C^{2}$-function. Let $A, B$ and $E$ be $C^{2}$-functions depending only on $r(t ; s)$ and $t$. Then, the identity

$$
\begin{aligned}
& \left(u_{t t}-\Delta u\right)\left(A u_{t}+B u_{r(t ; s)}+E u\right) \\
& \quad=F_{t}(u, t ; s)+\nabla \cdot G(u, t ; s)+H(u, t ; s)
\end{aligned}
$$

holds, where $F_{t}=\frac{\partial}{\partial t} F, G=\left(G_{1}, G_{2}, G_{3}\right)$ and

$$
\begin{aligned}
F(u, t ; s)= & \frac{1}{2} A\left(u_{t}^{2}+|\nabla u|^{2}\right)+u_{t}\left(B u_{r(t ; s)}+E u\right)-\frac{1}{2} E_{t} u^{2} \\
G_{j}(u, t ; s)= & -u_{j}\left(A u_{t}+B u_{r(t ; s)}+E u\right)+\frac{1}{2} z_{j}(x, t ; s) B\left(|\nabla u|^{2}-u_{t}^{2}\right)+\frac{1}{2} E_{j} u^{2} \\
H(u, t ; s)= & \frac{1}{2}\left(B_{r(t ; s)}-A_{t}+\frac{2 B}{r(t ; s)}-2 E\right) u_{t}^{2} \\
& +\frac{1}{2}\left(B_{r(t, s)}-A_{t}-\frac{2 B}{r(t ; s)}+2 E\right) u_{r(t ; s)}^{2} \\
& +\frac{1}{2}\left(A_{t}+B_{r(t ; s)}-2 E\right)\left(u_{r(t ; s)}^{2}-|\nabla u|^{2}\right) \\
& +\left(A_{j}-\left(B z_{j}(x, t ; s)\right)_{t}\right) u_{j} u_{t}+\frac{1}{2}\left(E_{t t}-E_{j j}\right) u^{2}
\end{aligned}
$$

Proof. The proof is tedious but elementary, so we omit it.

THEOREM 1. Suppose that Assumptions (1) $\sim(4)$ are satisfied. Let $v=v(t ; s)$ be a $C^{2}$-solution of problem $(\mathrm{P} .1 ; s) \sim(\mathrm{P} .3 ; s)$ for fixed $s \geq 1$. Suppose that the support of the initial data $f(x ; s)$ and $g(t ; s)$ is contained in $\left\{x \mid x \in \mathscr{E}(s), r(0 ; s) \leq N(s+\gamma)^{\alpha}\right\}, N>1$. Let $0<\delta<\beta \leq 1$. Then, there exist constants $s_{0}=s_{0}(N, \delta)$ and $C$ (independent of $T$ and $s$ ) such that for $s \geq s_{0}$ 


$$
\begin{gathered}
E(v ; \infty, T, s) \leq C E(v ; \infty, 0, s) \\
\int_{0}^{T} \int_{\delta(t ; s)}\left\{(1+r(t ; s))^{-1-\delta}\left(v_{t}^{2}+|\nabla v|^{2}\right)+(1+r(t ; s))^{-3-\delta} v^{2}\right\} d x d t \\
\leq C E(v ; \infty, 0, s) .
\end{gathered}
$$

Here $\alpha, \beta$ and $\gamma$ are the constants introduced in $\S 1$ and $E(;,$,$) is the$ notation defined by (1.7).

For the proof of Theorem 1, we have to prepare several lemmas. First, as $A, B$ and $E$ in Proposition 1, we take the following functions:

$$
A=1, \quad B=\zeta(r(t ; s)), \quad E=\zeta(r(t ; s)) r(t ; s)^{-1},
$$

where $\zeta(r)=\sigma-(\rho+r)^{-\delta}, 0<\delta<1,0<\sigma_{0}<\sigma<1$. Furthermore, we take $\rho=\rho(\delta)$ so large that for $r \geq 0$

$$
\zeta(r) \geq \sigma_{0} \quad \text { and } \quad \zeta(r) \frac{1}{r}-\zeta^{\prime}(r) \geq 0 .
$$

The following three lemmas are verified with a slight modification of the proof of Lemmas $1 \sim 3$ in [2].

LeMma 2.2. Let $A, B$ and $E$ be as defined by (2.7). Then, $F, G$ and $H$ in Proposition 1 are expressed as follows:

$$
\begin{aligned}
F(u ; t ; s)= & \frac{1}{2}\left(u_{t}^{2}+|\nabla u|^{2}\right)+\zeta(r(t ; s)) u_{t}\left(u_{r(t ; s)}+r(t ; s)^{-1} u\right) \\
& -\frac{1}{2}\left(\zeta(r(t ; s)) r(t ; s)^{-1}\right)_{t} u^{2}, \\
G_{j}(u, t ; s)= & -u_{j}\left(u_{t}+\zeta(r(t ; s)) u_{r(t ; s)}+\zeta(r(t ; s)) r(t ; s)^{-1} u\right) \\
& +\frac{1}{2} z_{j}(x, t ; s) \zeta(r(t ; s))\left(|\nabla u|^{2}-u_{t}^{2}\right) \\
& +\frac{1}{2} z_{j}(x, t ; s) r(t ; s)^{-1}\left(\zeta^{\prime}(r(t ; s))-\zeta(r(t ; s)) r(t ; s)^{-1}\right) u^{2}, \\
H(u, t ; s)= & \frac{1}{2} \zeta^{\prime}(r(t ; s))\left(u_{t}^{2}+|\nabla u|^{2}\right) \\
& +\left(\zeta(r(t ; s)) r(t ; s)^{-1}-\zeta^{\prime}(r(t ; s))\right)\left(|\nabla u|^{2}-u_{r(t ; s)}^{2}\right) \\
& -\left(\zeta\left(r(t ; s) z_{j}(x, t ; s)\right)_{t} u_{j} u_{t}\right. \\
& +\frac{1}{2}\left(\left(\zeta\left(r(t ; s) r(t ; s)^{-1}\right)_{t t}-\zeta^{\prime \prime}(r(t ; s)) r(t ; s)^{-1}\right) u^{2} .\right.
\end{aligned}
$$

Furthermore, it holds that

$$
\begin{aligned}
& \mid\left(\zeta\left(r(t ; s) z_{j}(x, t ; s)\right)_{t} \mid \leq C(1+r(t ; s))^{-1}(t+s+1)^{-\beta}\right. \\
& \left(\zeta(r(t ; s)) r(t ; s)^{-1}\right)_{t t}-\zeta^{\prime \prime}(r(t ; s)) r(t ; s)^{-1} \\
& \geq r(t ; s)^{-1}\left(\delta(1+\delta)(\rho+r(t ; s))^{-2-\delta}\right. \\
& -C\left((1+r(t ; s))^{-2-\delta}(t+s+1)^{-2 \beta}\right. \\
& \left.\left.+(1+r(t ; s))^{-1}(t+s+1)^{-1-\beta}\right)\right)
\end{aligned}
$$


for $r(t ; s) \geq \gamma_{0}, \gamma_{0}$ being the constant introduced in (1.6), where $C$ is a constant depending only on $\gamma_{0}$ and $\delta$.

Proof. We have only to insert $A, B$ and $E$ defined by (2.7) into the expression of $F, G$ and $H$. (2.9) and (2.10) follow from Lemma 2.1 and the definition of $\zeta(r)$ if we note that $\zeta^{\prime \prime}(r)=-\delta(1+\delta)(\rho+r)^{-2-\delta}$.

Lemma 2.3. Let $A, B$ and $E$ be as defined by (2.7). If we use the notation:

$$
w_{j}=u_{j}+z_{j}(x, t ; s) r(t ; s)^{-1} u \quad \text { and } \quad w_{z}=z_{j}(x, t ; s) w_{j}
$$

then $F(u, t ; s)$ is expressed in the following way:

$$
F(u, t ; s)=\varphi_{1}(u, t ; s)+\varphi_{2}(u, t ; s)+\varphi_{3}(u, t ; s)+\varphi_{4}(u, t ; s),
$$

where

$$
\begin{aligned}
& \varphi_{1}(u, t ; s)=\frac{1}{2}(1-\zeta(r(t ; s)))\left(u_{t}^{2}+|\nabla u|^{2}\right) \\
& \varphi_{2}(u, t ; s)=\frac{1}{2} \zeta(r(t ; s))\left(u_{t}^{2}+|w|^{2}+2 w_{z} u_{t}\right) \\
& \varphi_{3}(u, t ; s)=-\frac{1}{2}\left(\zeta(r(t ; s)) r(t ; s)^{-1} z_{j}(x, t ; s) u^{2}\right)_{j} \\
& \varphi_{4}(u, t ; s)=\frac{1}{2}\left(\zeta^{\prime}(r(t ; s)) r(t ; s)^{-1}-\left(\zeta(r(t ; s)) r(t ; s)^{-1}\right)_{t}\right) u^{2}
\end{aligned}
$$

Here we should note that $\varphi_{2}(u, t ; s) \geq 0$ and that

$$
\varphi_{1}(u, t ; s) \geq \frac{1}{2}(1-\sigma)\left(u_{t}^{2}+|\nabla u|^{2}\right),
$$

since $\zeta(r) \leq \sigma . \quad$ Furthermore, for $r(t ; s) \geq \gamma_{0}$

$$
\begin{aligned}
\varphi_{4}(u, t ; s) \geq & \frac{1}{2} r(t ; s)^{-1}(\rho+r(t ; s))^{-1}\left(\delta(\rho+r(t ; s))^{-\delta}\right. \\
& \left.-C(t+s+1)^{-\beta}\right) u^{2}
\end{aligned}
$$

with $C$ depending only on $\gamma_{0}$ and $\delta$.

Proof. The proof is done by a direct calculation and (2.12) follows from Lemma 2.1 if we note that $\zeta^{\prime}(r)=\delta(\rho+r)^{-1-\delta}$.

Lemma 2.4. Let $A, B$ and $E$ be as above. If $u=0$ on $\Sigma_{s}$, then

$$
n_{t} F(u, t ; s)+n_{j} G_{j}(u, t ; s)=\frac{1}{2}\left(n_{t}^{2}-\left|n_{x}\right|^{2}\right)\left(n_{t}+\zeta(r(t ; s)) n_{r(t ; s)}\right)\left|\frac{\partial u}{\partial n}\right|^{2}
$$

for $(x, t) \in \Sigma_{s}$, where $n_{r(t ; s)}=n_{j} z_{j}(x, t ; s)$.

Proof. Since $u=0$ on $\Sigma_{s}$, all the tangential derivatives of $u$ also 
vanish there, so that on $\Sigma_{s}$

$$
u_{t}=n_{t} \frac{\partial u}{\partial n}, \quad u_{j}=n_{j} \frac{\partial u}{\partial n}, \quad u_{r(t ; s)}=n_{r(t ; s)} \frac{\partial u}{\partial n} .
$$

Hence, if we have only to insert these expressions into $n_{t} F(u, t ; s)$ $+n_{j} G_{j}(u, t ; s)$, we obtain the desired result.

Now, we shall prove Theorem 1 with the aid of Lemmas $2.2 \sim 2.4$.

Proof of Theorem 1. We integrate the identity (2.6) with $A, B$ and $E$ defined by $(2.7)$ and $u=v(t ; s)$ over $\mathscr{E}_{s}(0, T)$. Then, we have

$$
\begin{aligned}
\int_{\delta(T ; s)} F(v, T ; s) d x & +\int_{0}^{T} \int_{\Sigma(t ; s)}\left(n_{t} F(v, t ; s)+n_{j} G_{j}(v, t ; s)\right) d S d t \\
& +\int_{0}^{T} \int_{\delta(t ; s)} H(v, t ; s) d x d t=\int_{\delta(0 ; s)} F(v, 0 ; s) d x .
\end{aligned}
$$

By (2.8), (1.3) and Assumption (3),

$$
n_{t}+\zeta(r(t ; s)) n_{r(t ; s)} \leq n_{t}+\sigma_{0} n_{r(t ; s)} \leq 0
$$

on $\Sigma_{s}$, so that it follows from Assumption (1) and Lemma 2.4 that the second term on the left side of (2.13) is non-negative. Hence, this term can be thrown away.

Next we consider the first term and recall the expressions of $\varphi_{j}(u, t ; s)$, $j=1, \cdots, 4$, in Lemma 2.3. From the condition on the support of the initial data $f(x ; s)$ and $g(x ; s)$, we see by Assumption (1) and by the argument of the dependence of domain that $v(t ; s)=0$ for $|x-a(s)|$ $\geq t+N(s+\gamma)^{\alpha}$ i.e. $r(0 ; s) \geq t+N(s+\gamma)^{\alpha}, 0 \leq \alpha<1$. Consequently, taking account of (2.2) in Lemma 2.1, we have that $v(t ; s)=0$ for $r(t ; s)$ $\geq(1+\mu) t+N(s+\gamma)^{\alpha}$, since $r(t ; s) \leq r(0 ; s)+\mu t$. Therefore, by (2.12) and the condition $0<\delta<\beta$, there exists a constant $s_{1}=s_{1}(N, \delta)$ such that for $s \geq s_{1}, \varphi_{4}(v, t ; s) \geq 0$. From this fact and (2.11), we conclude that

$$
\int_{\sigma(T ; s)} F(v, T ; s) d x \geq \frac{1}{2}(1-\sigma) E(v ; \infty, T, s) .
$$

Finally we consider the third term on the left side of (2.13). Recalling the expression of $H(u, t ; s)$ in Lemma 2.2, we see that the second term is non-negative and that the first term is estimated from below by

$$
\frac{1}{2} \delta(\rho+r(t ; s))^{-1-\delta}\left(v_{t}^{2}+|\nabla v|^{2}\right) .
$$


By (2.9) the third term is absorbed in the first term, if we take $s$ large enough and note that $\delta<\beta$. And also, in view of (2.10), the fourth term is dealt with by the above argument using the dependence of domain. Thus, there exists a constant $s_{0}=s_{0}(N, \delta), s_{0} \geq s_{1}$, such that for $s \geq s_{0}$

$$
H(v, t ; s) \geq C_{1}(1+r(t ; s))^{-1-\delta}\left(v_{t}^{2}+|\nabla v|^{2}\right)+C_{2}(1+r(t ; s))^{-3-\delta} v^{2} .
$$

We shall estimate the term on the right side of (2.13). By use of the estimate (Poincaré's inequality):

$$
\int_{\delta(0 ; s)} r(0 ; s)^{-2} v(0 ; s)^{2} d x \leq C \int_{\delta(0 ; s)}|\nabla v(0 ; s)|^{2} d x
$$

for $C$ independent of $s$, it is easy to see that

$$
\int_{\delta(0 ; s)} F(v, 0 ; s) d x \leq C E(v ; \infty, 0, s) .
$$

Thus, combining all the investigation given above, we obtain the desired estimate.

THEOREM 2. Suppose that the same assumptions as in Theorem 1 are satisfied. Then,

$$
\begin{gathered}
\int_{0}^{T} \int_{\Sigma(t ; s)}(r(t ; s)+t)\left(n_{t}^{2}-\left|n_{x}\right|^{2}\right) n_{r(t ; s)}\left|\frac{\partial u}{\partial n}\right|^{2} d S d t \\
\leq C\left(s^{\alpha}+T^{1+\delta-\beta}\right) E(v ; \infty, 0, s)
\end{gathered}
$$

for $s \geq s_{0}, s_{0}$ being the constant introduced in Theorem 1 .

For the proof of Theorem 2, we take as $A, B$ and $E$ in Proposition 1 the following functions:

$$
A=B=r(t ; s)+t, \quad E=(r(t ; s)+t) r(t ; s)^{-1} .
$$

Then, the next lemma corresponding to Lemmas $2.2 \sim 2.4$ holds.

LEMMA 2.5. Let $A, B$ and $E$ be as given by (2.14). Then, the following statements hold.

(i) $F(u, t ; s)$ can be expressed as follows:

$$
F(u, t ; s)=\psi_{1}(u, t ; s)+\psi_{2}(u, t ; s)+\psi_{3}(u, t ; s),
$$

where 


$$
\begin{aligned}
\psi_{1}(u, t ; s)= & \frac{1}{2} A\left\{u_{t}^{2}+2 u_{t}\left(u_{r(t ; s)}+\frac{E}{A} u\right)+\left(u_{r(t ; s)}+\frac{E}{A} u\right)^{2}\right. \\
& \left.+\left(|\nabla u|^{2}-u_{r(t ; s)}^{2}\right)\right\}, \\
\psi_{2}(u, t ; s)= & -\frac{1}{2}\left(E z_{j}(x, t ; s) u^{2}\right)_{j}, \\
\psi_{3}(u, t ; s)= & -\frac{1}{2} t\left(r(t ; s)^{-1}\right)_{t} u^{2} .
\end{aligned}
$$

Furthermore

$$
\left|\psi_{3}(u, t ; s)\right| \leq C(t+1)^{1-\beta} r(t ; s)^{-2} u^{2} .
$$

(ii) If $u=0$ on $\Sigma_{s}$, and if Assumptions (1) $\sim$ (3) are satisfied, then

$$
\begin{aligned}
n_{t} F(u, t ; s)+n_{j} G_{j}(u, t ; s) & =\frac{1}{2} A\left(n_{t}^{2}-\left|n_{x}\right|^{2}\right)\left(n_{t}+n_{r(t ; s)}\right)\left|\frac{\partial u}{\partial n}\right|^{2} \\
& \geq \frac{1}{2}\left(1-\sigma_{0}\right) A\left(n_{t}^{2}-\left|n_{x}\right|^{2}\right) n_{r(t ; s)}\left|\frac{\partial u}{\partial n}\right|^{2}, \quad \sigma_{0}<1 .
\end{aligned}
$$

(iii) $H(u, t ; s)$ can be estimated from below in the following way:

$$
H(u, t ; s) \geq-H_{1}(u, t ; s),
$$

where

$$
\begin{aligned}
H_{1}(u, t ; s)= & C_{1}(r(t ; s)+t) r(t ; s)^{-1}(t+s+1)^{-\beta}\left(u_{t}^{2}+|\nabla u|^{2}\right) \\
& +C_{2} r(t ; s)^{-2}(t+s+1)^{-\beta}\left(1+\operatorname{tr}(t ; s)^{-1}\right) u^{2}
\end{aligned}
$$

with $C_{1}$ and $C_{2}$ independent of $s$.

Proof. (i) is verified by a direct calculation and (2.15) readily follows from Lemma 2.1. The proof of (ii) is the same as that of Lemma 2.4 and the estimate from below follows from Assumptions (1) (3). (iii) is proved as follows: Inserting $A, B$ and $E$ defined by (2.14) into the expression of $H(u, t ; s)$, we have

$$
\begin{aligned}
H(u, t ; s)= & -\frac{1}{2} r_{t}(t ; s)\left(u_{t}^{2}+|\nabla u|^{2}\right)+t r(t ; s)^{-1}\left(|\nabla u|^{2}-u_{r(t ; s)}^{2}\right) \\
& -\left(r_{t}(t ; s) z_{j}(x, t ; s)+(r(t ; s)+t) z_{j t}(x, t ; s)\right) u_{j} u_{t} \\
& -\frac{1}{2} r(t ; s)^{-2}\left(2 r_{t}(t ; s)+t r_{t t}(t ; s)-2 t r(t ; s)^{-1} r_{t}(t ; s)^{2}\right) u^{2}
\end{aligned}
$$

The second term is non-negative, so that it can be thrown away. The remaining terms are estimated with the aid of Lemma 2.1 and we obtain the desired result.

Proof of Theorem 2. As in the proof of Theorem 1, we integrate 
the identity (2.6) with $A, B$ and $E$ defined by (2.14) and $u=v(t ; s)$ over $\mathscr{E}_{s}(0, T)$. Then, we have

$$
\begin{gathered}
\int_{0}^{T} \int_{\Sigma(t ; s)}\left(n_{t} F(v, t ; s)+n_{j} G_{j}(v, t ; s)\right) d S d t=\int_{\delta(0 ; s)} F(v, 0 ; s) d x \\
-\int_{\delta(T ; s)} F(v, T ; s) d x-\int_{0}^{T} \int_{\delta(t ; s)} H(v, t ; s) d x d t .
\end{gathered}
$$

By (ii) of Lemma 2.5, the left side is estimated from below by

$$
\frac{1}{2}\left(1-\sigma_{0}\right) \int_{0}^{T} \int_{\Sigma(t ; s)}(r(t ; s)+t)\left(n_{t}^{2}-\left|n_{x}\right|^{2}\right) n_{r(t ; s)}\left|\frac{\partial u}{\partial n}\right|^{2} d S d t .
$$

We shall estimate the three terms appearing on the right side. By the condition on the support of the initial data, it is easily seen that

$$
\int_{\varepsilon(0 ; s)} F(v, 0 ; s) d x \leq C s^{\alpha} E(v ; \infty, 0, s) .
$$

Next we consider the second term. Recall the expression of $\psi_{j}(u, t ; s)$, $j=1 \sim 3$, in (i) of Lemma 2.5. Then, since $\psi_{1}(v, T ; s) \geq 0$,

$$
-\int_{\delta(T ; s)} F(v, T ; s) d x \leq-\int_{\delta(T ; s)} \psi_{3}(v, T ; s) d x .
$$

Furthermore, by use of (2.15) and the Poincaré inequality, we obtain

$$
-\int_{\delta(T ; s)} F(v, T ; s) d x \leq C(T+1)^{1-\beta} E(v ; \infty, T, s),
$$

so that, in view of Theorem 1 ,

$$
-\int_{\delta(T ; s)} F(v, T ; s) d x \leq C(T+1)^{1-\beta} E(v ; \infty, 0, s) .
$$

Finally we deal with the third term. By (iii) of Lemma 2.5,

$$
-\int_{0}^{T} \int_{\delta(t ; s)} H(v, t ; s) d x d t \leq \int_{0}^{T} \int_{\delta(t ; s)} H_{1}(v, t ; s) d x d t .
$$

Furthermore we have shown in the proof of Theorem 1 that $v(t ; s)=0$ for $r(t ; s) \geq(1+\mu) t+N(s+\gamma)^{\alpha}$. Consequently,

$$
\begin{aligned}
H_{1}(v, t ; s) \leq & C_{3}\left(T+s^{\alpha}\right)^{1+\delta-\beta}\left\{(1+r(t ; s))^{-1-\delta}\left(v_{t}^{2}+|\nabla v|^{2}\right)\right. \\
& \left.+(1+r(t ; s))^{-3-\delta} v^{2}\right\} .
\end{aligned}
$$

We combine this estimate with Theorem 1 to obtain 


$$
-\int_{0}^{T} \int_{\odot(t ; s)} H(v, t ; s) d x d t \leq C\left(T+s^{\alpha}\right)^{1+\delta-\beta} E(v ; \infty, 0, s) .
$$

Thus the proof is complete.

The next theorem gives the uniform decay of the local enery.

THEOREM 3. Suppose that the same assumptions as in Theorem 1 are satisfied. Then,

$$
E\left(v ; \frac{1}{2} T, T, s\right) \leq C T^{-2}\left(s^{2 \alpha}+s^{\alpha} T+T^{2+\delta-\beta}\right) E(v ; \infty, 0, s)
$$

for $s \geq s_{0}, s_{0}$ being the constant introduced in Theorem 1 .

For the proof of this theorem, we set

$$
A=r(t ; s)^{2}+t^{2}, \quad B=2 \operatorname{tr}(t ; s), \quad E=2 t .
$$

Lemma 2.6. Let $A, B$ and $E$ be as given by (2.16). Then, the following statements hold.

(i) $F(u, t ; s)$ is expressed as $F(u, t ; s)=F_{1}(u, t ; s)+F_{2}(u, t ; s)$, where

$$
\begin{aligned}
F_{1}(u, t ; s)= & \frac{1}{2} A\left(u_{t}^{2}+|\nabla u|^{2}\right)+u_{t}\left(B u_{r(t ; s)}+E u\right) \\
& +A r(t ; s)^{-2}\left(r(t ; s) u_{r(t ; s)} u+\frac{1}{2} u^{2}\right), \\
F_{2}(u, t ; s)= & -\frac{1}{2}\left(A r(t ; s)^{-1} z_{j}(x, t ; s) u^{2}\right)_{j} .
\end{aligned}
$$

Furthermore, $F_{1}(u, t ; s) \geq 0$ and for $r(t ; s) \leq \frac{1}{2} t$

$$
F_{1}(u, t ; s) \geq \frac{1}{8} t^{2}\left\{u_{t}^{2}+|\nabla u|^{2}+\left(r(t ; s)^{-1} z_{j}(x, t ; s) u^{2}\right)_{j}\right\} .
$$

(ii) If $u=0$ on $\Sigma_{s}$ and if Assumptions (1) $\sim$ (3) are satisfied, then

$$
\begin{aligned}
n_{t} F(u, t ; s)+n_{j} G_{j}(u, t ; s)= & \frac{1}{2}\left(n_{t}^{2}-\left|n_{x}\right|^{2}\right)\left(A n_{t}+B n_{r(t ; s)}\right)\left|\frac{\partial u}{\partial n}\right|^{2} \\
& \geq-\frac{1}{2} \sigma_{0} A\left(n_{t}^{2}-\left|n_{x}\right|^{2}\right) n_{r(t ; s)}\left|\frac{\partial u}{\partial n}\right|^{2} .
\end{aligned}
$$

(iii) $H(u, t ; s)$ satisfies the estimate:

$$
H(u, t ; s) \leq C(t+s+1)^{-\beta}(r(t ; s)+t)\left(u_{t}^{2}+|\nabla u|^{2}\right) .
$$

Proof. (i) is verified exactly in the same way as in Lax and Phillips [4], Appendix $3^{0)}$. The proof of (ii) is the same as that of Lemma 2.4 and the estimate from below follows from Assumptions (1) (3). (iii) is proved by a direct calculation with the aid of Lemma 2.1.

0) We use the identity: $-u^{2}=A r^{-2}\left(r u_{r} u+\frac{1}{2} u^{2}\right)-\frac{1}{2}\left(A r^{-1} z_{j}(x, t ; s) u^{2}\right)_{j}, r=r(t ; s)$. 
Proof of Theorem 3. The proof is very similar to that of Theorems. 1 and 2. Integrating the identity (2.6) with $A, B$ and $E$ defined by (2.16), and $u=v(t ; s)$, we have

$$
\begin{aligned}
\int_{\ell(T ; s)} F(v, T ; s) d x= & \int_{\delta(0 ; s)} F(v, 0 ; s) d x \\
& -\int_{0}^{T} \int_{\Sigma(t ; s)}\left(n_{t} F(v, t ; s)+n_{j} G_{j}(v, t ; s)\right) d S d t \\
& -\int_{0}^{T} \int_{\delta(t ; s)} H(v, t ; s) d x d t .
\end{aligned}
$$

We shall estimate the three terms on the right side of (2.17). First, by the condition on the support of the initial data, we easily have

$$
\int_{\delta(0 ; s)} F(v, 0 ; s) d x \leq C s^{2 \alpha} E(v ; \infty, 0, s) .
$$

For the second term, using (ii) of Lemma 2.6, we see that it is majorized by

$$
\frac{1}{2} \sigma_{0} \int_{0}^{T} \int_{\Sigma(t ; s)}\left(r(t ; s)^{2}+t^{2}\right)\left(n_{t}^{2}-\left|n_{x}\right|^{2}\right) n_{r(t ; s)}\left|\frac{\partial u}{\partial n}\right|^{2} d S d t
$$

Furthermore, since it follows from Assumption (4) that on $\Sigma(t ; s)$

$$
r(t ; s) \leq C(t+s)^{\alpha},
$$

we combine this fact with Theorem 2 to obtain that the second term is majorized by

$$
C\left(s^{2 \alpha}+s^{\alpha} T+T^{2+\delta-\beta}\right) E(v ; \infty, 0, s) .
$$

We deal with the third term. By use of the fact that $v(t ; s)=0$ for $r(t ; s) \geq(1+\mu) t+N(s+\gamma)^{\alpha}$, it follows from (iii) of Lemma 2.6 that

$$
H(v, t ; s) \leq C\left(T+s^{\alpha}\right)^{2+\delta-\beta}(1+r(t ; s))^{-1-\delta}\left(v_{t}^{2}+|\nabla v|^{2}\right)
$$

for $0 \leq t \leq T$. Hence we combine this estimate with Theorem 1 to conclude that

$$
\int_{0}^{T} \int_{\delta(t ; s)} H(v, t ; s) d x d t \leq C\left(T+s^{\alpha}\right)^{2+\delta-\beta} E(v ; \infty, 0, s) .
$$

Obviously, by (i) of Lemma 2.6, the left side of (2.17) is estimated from 
below by

$$
\frac{1}{3} T^{2} E\left(v ; \frac{1}{2} T, T, s\right) .
$$

Thus the proof is complete.

We consider the following transformation of variables:

$$
y=x-a(t), \quad \tau=t .
$$

We denote by $\Omega(\tau)$ and $B(\tau), \tau \geq 0$, the domain transformed by (2.19) of $\mathscr{E}(t)$ and $\mathcal{O}(t)$, respectively. Let

$$
\Omega=\bigcup_{0<\tau<\infty} \Omega(\tau)
$$

and for each fixed $s \geq 0$, the notations $\Omega_{s}$ and $\Omega(T ; s)$ are introduced in the same way as $\mathscr{E}_{s}$ and $\mathscr{E}(T ; s)$, respectively. Furthermore, by Assumption (4), it holds that for each $\tau \geq 0$

$$
\left\{y \| y \mid \leq \gamma_{0}\right\} \subset B(\tau) \subset\left\{\left(y \| y \mid \leq(\tau+\gamma)^{\alpha}\right\} .\right.
$$

Now we transform the problem $(\mathrm{P} .1 ; s) \sim(\mathrm{P} .3 ; s)$ by $(2.19)$ :

$(\mathrm{Q} .1 ; s)$

$$
\begin{array}{r}
V_{\tau \tau}-2 a_{j_{\tau}}(\tau ; s) V_{\tau j}+a_{i \tau}(\tau ; s) a_{j_{\tau}}(\tau ; s) V_{i j} \\
-V_{j j}-a_{j \tau \tau}(\tau ; s) V_{j}=0 \quad \text { in } \Omega_{s}
\end{array}
$$

$(\mathrm{Q} .2 ; s) \quad V=0$ on $\partial \Omega(\tau ; s), \partial \Omega(\tau ; s)$ being the boundary of $\Omega(\tau ; s)$

$(\mathrm{Q} .3 ; s) \quad V(y, 0 ; s)=F(y ; s), \quad V_{\tau}(y, 0 ; s)=G(y ; s) \quad$ on $\Omega(0 ; s)$,

where $a_{j}(\tau ; s)=a_{j}(\tau+s)$ and $a_{j_{\tau}}(\tau ; s)=\frac{d}{d \tau} a_{j}(\tau ; s)$, while by (1.1), the operator $a_{i \tau}(\tau ; s) a_{j_{\tau}}(\tau ; s) \frac{\partial^{2}}{\partial y_{i} \partial y_{j}}-\Delta$ is uniformly elliptic. We denote by $V(\tau ; s)=V(y, \tau ; s)$ a solution of problem $(\mathrm{Q} .1 ; s) \sim(\mathrm{Q} .3 ; s)$ and $V(\tau ; s)$ is represented through the solution $v(t ; s)=v(x, t ; s)$ of problem $(\mathrm{P} .1 ; s)$ $\sim(\mathrm{P} .3 ; s)$ as $V(y, \tau ; s)=v(y+a(\tau ; s), \tau ; s)$. For this $V(\tau ; s)$, we define the local energy measured over $\mathscr{D}(h ; s)=\{y|y \in \Omega(T ; s)| y \mid, \leq h\}, 0<h$ $\leq \infty$, at $\tau=T$ by

$$
E_{0}(V ; h, T, s)=\int_{\mathscr{Q}(h ; s)}\left(\left|V_{\tau}(T ; s)\right|^{2}+|\nabla V(T ; s)|^{2}\right) d y .
$$

LEMMA 2.7. Let $v(t ; s)$ and $V(\tau ; s)$ be solutions of problems $(\mathrm{P} .1 ; s)$ $\sim(\mathrm{P} .3 ; s)$ and $(\mathrm{Q} .1 ; s) \sim(\mathrm{Q} .3 ; s)$, respectively. Then there exist con- 
stants $C_{1}$ and $C_{2}$ independent of $h, T$ and $s$ such that

$$
C_{1} E(v ; h, T, s) \leq E_{0}(V ; h, T, s) \leq C_{2} E(v ; h, T, s) .
$$

The next theorem is an immediate consequence of Theorem 3 and Lemma 2.7.

THEOREM 4. Suppose that Assumptions (1) $\sim(4)$ are satisfied. Let $V(\tau ; s)$ be a $C^{2}$-solution of problem $(\mathrm{Q} .1 ; s) \sim(\mathrm{Q} .3 ; s)$ with the initial data $\quad F(y ; s) \quad\left(C^{\infty}(\Omega(0 ; s)) \cap H_{0}^{1}(\Omega(0 ; s))\right) \quad$ and $G(y ; s) \quad\left(C^{\infty}(\Omega(0 ; s))\right.$ $\left.\cap L^{2}(\Omega(0 ; s))\right)$. Suppose that the support of $F(y ; s)$ and $G(y ; s)$ is contained in $\left\{y|| y \mid \leq N(s+\gamma)^{\alpha}\right\}$. Let $0<\delta<\beta \leq 1$. Then there exist constants $s_{0}=s_{0}(N, \delta)$ and $C$ (independent of $T$ and $s$ ) such that for $s \geq s_{0}$

$$
E_{0}\left(V ; \frac{1}{2} T, T, s\right) \leq C T^{-2}\left(s^{2 \alpha}+s^{\alpha} T+T^{2+\delta-\beta}\right) E_{0}(V ; \infty, 0, s) .
$$

Remark. Theorem 4 is valid also for a weak solution with the initial data $F(y ; s)\left(\in H_{0}^{1}(\Omega(0 ; s))\right)$ and $G(y ; s)\left(\in L^{2}(\Omega(0 ; s))\right)$ verifying the condition for the support stated above.

Theorem 4 may be directly obtained by considering the transformed equation $(\mathrm{Q} .1 ; s)$ from the beginning. However, a calculation then will be more complicated because of the appearance of the term $V_{j \tau}$.

\section{§3. Proof of main result}

\subsection{Huyghen's Principle}

We denote by $L(s), s \geq 0$, the operator

$$
\begin{aligned}
L(s) W= & W_{\tau \tau}-2 a_{j \tau}(\tau ; s) W_{j \tau} \\
& +a_{i \tau}(\tau ; s) a_{j_{\tau}}(\tau ; s) W_{i j}-W_{j j}-a_{j \tau \tau}(\tau ; s) W_{j} .
\end{aligned}
$$

We consider the equation

$$
L(s) W=0 \quad \text { in } R^{3} \times(0, \infty)
$$

with the initial data $W(y, 0 ; s)\left(\in H^{1}\left(R^{3}\right)\right)$ and $W_{\tau}(y, 0 ; s)\left(\in L^{2}\left(R^{3}\right)\right)$. Then, the (weak) solution $W(\tau ; s)=W(y, \tau ; s)$ is expressed through the free space solution $w(x, t), \square w=0$, as follows:

$$
W(y, \tau ; s)=w(y+a(\tau ; s), \tau),
$$

where the initial data $w(x, 0)$ and $w_{t}(x, 0)$ are given by 


$$
\begin{aligned}
w(x, 0) & =W(x-a(0 ; s), 0, ; s), \\
w_{t}(x, 0) & =W_{z}(x-a(0 ; s), 0 ; s)-a_{j_{z}}(0 ; s) W_{j}(x-a(0 ; s), 0 ; s) .
\end{aligned}
$$

For given $\left(x_{0}, t_{0}\right)$ and fixed $t, t \leq t_{0}$, it follows from Huyghen's principle that the value $w\left(x_{0}, t_{0}\right)$ is determined only by the value of $w(x, t)$ on the sphere $\left|x-x_{0}\right|=t_{0}-t$. Therefore, using the relation (3.2), we see that for given $\left(y_{0}, \tau_{0}\right)$ and fixed $\tau, \tau \leq \tau_{0}$, the value $W\left(y_{0}, \tau_{0} ; s\right)$ is determined only by the value of $W(y, \tau ; s)$ on the sphere $\left|y+a(\tau ; s)-y_{0}-a\left(\tau_{0} ; s\right)\right|$ $=\tau_{0}-\tau$. Furthermore, if the support of the initial data $W(y, 0 ; s)$ and $W_{\tau}(y, 0 ; s)$ is contained in $|y| \leq K$, then the support of $w(x, 0)$ and $w_{t}(x, 0)$ is contained in $|x-a(0 ; s)| \leq K$. We again apply Huyghen's principle to $w(x, t)$ to conclude that $w(x, t)=0$ for $|x-a(0 ; s)| \leq t-K$, so that $W(y, \tau ; s)=0$ for $|y+a(\tau ; s)-a(0 ; s)| \leq \tau-K$.

Summing up the above investigation, we have the following proposition.

Proposition 2. Let $W(\tau ; s)=W(y, \tau ; s)$ be a (weak) solution of problem $(\mathrm{Q} ; s)$. Then the following statements hold.

(i) For given $\left(y_{0}, \tau_{0}\right)$ and fixed $\tau, \tau \leq \tau_{0}$, the value $W\left(y_{0}, \tau_{0} ; s\right)$ is determined only by the value of $W(y, \tau ; s)$ on the sphere $\mid y+a(\tau ; s)-y_{0}$ $-a\left(\tau_{0} ; s\right) \mid=\tau_{0}-\tau$.

(ii) The backward cone with vertex $\left(y_{0}, \tau_{0}\right)$ expressed by $\left|y-y_{0}\right|=(1-\mu)$ $\left(\tau_{0}-\tau\right)$ is contained in the interior of the backward cone with the same vertex expressed by $\left|y+a(\tau ; s)-y_{0}-a\left(\tau_{0} ; s\right)\right|=\tau_{0}-\tau$.

(iii) If the initial data $W(y, 0 ; s)$ and $W_{\tau}(y, 0 ; s)$ have compact support contained in $|y| \leq K$, then $W(y, \tau ; s)=0$ for $|y+a(\tau ; s)-a(0 ; s)| \leq \tau-K$, so that $W(y, \tau ; s)=0$ for $|y| \leq(1-\mu) \tau-K$.

Here the constant $\mu$ is as introduced in (1.1).

Proof. (i) has been proved above. (ii) is verified with the aid of (2.2) in Lemma 2.1. (iii) follows from (ii).

For the solution $W(\tau ; s)$ of problem $(\mathrm{Q} ; s)$, we define the local energy measured over $|y|<h, 0<h \leq \infty$, at $\tau=T$ by

$$
\hat{E}_{0}(W ; h, T, s)=\int_{|y|<h}\left(\left|W_{\tau}(T ; s)\right|^{2}+|\nabla W(T ; s)|^{2}\right) d y .
$$

Proposition 3. Let $W(\tau ; s)$ be a solution of problem $(\mathrm{Q} ; s)$. Then, there exists a constant $C$ independent of $s, T$ and $h$ such that 


$$
\hat{E}_{0}(W ; h, T, s) \leq C \hat{E}_{0}(W ; h+(1+\mu) T, 0, s)
$$

This implies that the motion governed by $(\mathrm{Q} ; s)$ propagates at a speed less than $1+\mu$.

Proof. The assertion follows from relation (3.2) and the fact that the motion governed by the free space wave equation propagates at the speed one.

\subsection{Preliminary lemma}

We define several sequences, following the method in [6]. Let $0<\delta$ $<\beta \leq 1$ and let $0 \leq \alpha<1$. We put

$$
p \geq \alpha(1-\alpha)^{-1}
$$

so that

$$
p \geq \alpha(p+1)
$$

Let $\left\{T_{k}\right\}_{k=0}^{\infty}$ be the sequence given by

$$
T_{k}=k^{p} T,
$$

$T$ being large enough (determined below, Lemma 3.2) and let

$$
S_{k}=\sum_{m=0}^{k} T_{m}
$$

so that

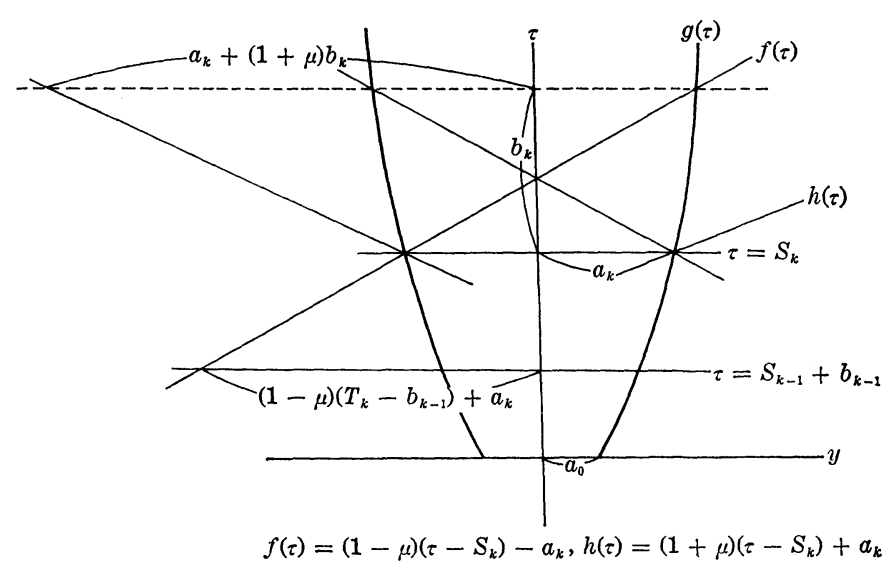

Fig. 1 


$$
S_{k} \leq C_{p} k^{p+1} T
$$

We put $g(\tau)=(\tau+\gamma)^{\alpha}, \gamma>1$, and define the sequence $\left\{a_{k}\right\}_{k=0}^{\infty}, a_{k}>1$, by

$$
a_{k}=g\left(S_{k}\right) \quad\left(a_{0}=\gamma^{\alpha}\right) .
$$

Furthermore, we define the sequence $\left\{b_{k}\right\}_{k=0}^{\infty}, b_{k}>0$, as follows :

(3.7) $b_{k}$ is a (unique) root of the equation: $(1-\mu) t-a_{k}=g\left(t+S_{k}\right)$ (Fig. 1).

LEMMA 3.1. There exists a constant $M$ independent of $k \geq 0$ and T such that

$$
a_{k} \leq b_{k} \leq M a_{k}
$$

Proof. The proof is obvious from Fig. 1.

LEMma 3.2. We can take $T$ so large that for $k \geq 1$

$$
\begin{gathered}
a_{k}+2(1+\mu) b_{k} \leq \frac{1}{2}\left(T_{k}-b_{k-1}\right), \\
a_{k-1}+(1+\mu) b_{k-1} \leq(1-\mu)\left(T_{k}-b_{k-1}\right)+a_{k}, \\
\frac{1}{2} k^{p} T \leq T_{k}-b_{k-1} .
\end{gathered}
$$

Proof. By definition and Lemma 3.1, $T_{k}=O\left(k^{p} T\right), a_{k}=O\left(k^{\alpha(p+1)} T^{\alpha}\right)$ and $b_{k}=O\left(k^{\alpha(p+1)} T^{\alpha}\right)$. Since $0 \leq \alpha<1$ and since $p \geq \alpha(p+1)$ by (3.4.1), we can find such a $T$.

\subsection{Proof of main theorem}

We shall prove Main Theorem stated in $\S 1$. To this end, we introduce the new notation: For $G \in L^{2}(\mathscr{D}), \mathscr{D}\left(\subset R^{3}\right)$ being an arbitrary domain, we define $\tilde{G}$ by $\tilde{G}=G$ in $\mathscr{D}$ and $\tilde{G}=0$ in $R^{3}-\mathscr{D}$.

LEMMA 3.3. Let $U(y, \tau)$ be a (weak) solution of problem $(\mathrm{Q} .1 ; 0)$ $\sim(\mathrm{Q} .2 ; 0)$ with the initial data $F\left(\in H_{0}^{1}(\Omega(0))\right)$ and $G\left(\in L^{2}(\Omega(0))\right)$ such that the support of $F$ and $G$ is contained in $\left\{y|y \in \Omega(0)| y \mid, \leq \gamma^{\alpha}\right\}, a_{0}=\gamma^{\alpha} .^{1)}$ Then, the solution $U$ may be written as

$$
U=R_{0}+F_{0},
$$

where $F_{0}$ is a solution (defined over the whole space) of $L(0) F_{0}=0$ with

1) This domain is not void by taking $r$ large enough if necessary. 
the initial data $\tilde{F}^{\prime}\left(\in H^{1}\left(R^{3}\right)\right)$ and $\tilde{G}, L(0)$ being the operator given by (3.1), and

$$
F_{0}=0 \quad \text { for }|y| \leq(1-\mu) \tau-a_{0},
$$

while $R_{0}$ is a solution of problem $\left(\mathrm{Q} .1 ; b_{0}\right) \sim\left(\mathrm{Q} .2 ; b_{0}\right)$ (that is, $R_{0}$ is a solution of problem $(\mathrm{Q} .1 ; 0) \sim(\mathrm{Q} .2 ; 0)$ for $\left.\tau>b_{0}\right)$ and has compact support of at most $|y| \leq a_{0}+(1+\mu) b_{0}$ at $\tau=b_{0}$. Furthermore

$$
E_{0}\left(R_{0} ; \infty, 0, b_{0}\right) \leq C\left(b_{0}\right) E_{0}(U ; \infty, 0,0) .
$$

Proof. The assertion for $F_{0}$ follows from (iii) in Proposition 2. By the definition of $b_{0}$ (see Fig. 1),

$$
F_{0}=0 \quad \text { in }|y| \leq(\tau+\gamma)^{\alpha}, \tau>b_{0},
$$

so that by (2.20) $F_{0}=0$ on $\bigcup_{b_{0}<\tau<\infty} \partial \Omega(\tau) \times\{\tau\}, \partial \Omega(\tau)$ being the boundary of $\Omega(\tau)$. This implies that $R_{0}$ is a solution of problem $\left(\mathrm{Q} .1 ; b_{0}\right) \sim\left(\mathrm{Q} .2 ; b_{0}\right)$. The second assertion for $R_{0}$ follows from Proposition $3 .^{2)} \quad(3.11)$ is verified as follows :

$$
E_{0}\left(R_{0} ; \infty, 0, b_{0}\right) \leq 2\left(E_{0}\left(F_{0} ; \infty, 0, b_{0}\right)+E_{0}\left(U ; \infty, 0, b_{0}\right)\right) .
$$

We claim that

$$
\begin{gathered}
E_{0}\left(F_{0}, \infty, 0, b_{0}\right) \leq C E_{0}(U ; \infty, 0,0) \\
E_{0}\left(U, \infty, 0, b_{0}\right) \leq C\left(b_{0}\right) E_{0}(U ; \infty, 0,0) .
\end{gathered}
$$

Recalling the notation $\hat{E}_{0}(;$, ,) given by (3.3) and using the property for $F_{0}$, we have

$$
E_{0}\left(F_{0} ; \infty, 0, b_{0}\right)=\hat{E}_{0}\left(F_{0} ; \infty, b_{0}, 0\right),
$$

whence (3.12) follows with the aid of Proposition 3, since the initial data of $F_{0}$ are $\tilde{F}$ and $\tilde{G}$. For the proof of (3.13), we give only a sketch. By an argument similar (more simple) to that given in the proof of Theorem $1^{3)}$ and by Lemma 2.7 , we easily have

$$
\begin{aligned}
& E_{0}\left(U ; \infty, b_{0}, 0\right)\left(=E_{0}\left(U ; \infty, 0, b_{0}\right)\right) \\
& \quad \leq C_{1} E_{0}(U ; \infty, 0,0)+C_{2}\left(b_{0}\right) \int_{0}^{b_{0}} E_{0}(U ; \infty, t, 0) d t .
\end{aligned}
$$

2) In Propositions 2 and 3, only the whole space solution was discussed. However, from the argument given there we see that these propositions are valid for the solution considered here.

3) We use the identity (2.6) with $A$ and $B$ defined by (2.7) and $E=0$. 
We have only to apply the well known Gronwall inequality to this estimate.

LEMMA 3.4. Suppose that the same assumptions in Lemma 3.3 are satisfied. Let $\left\{T_{k}\right\}_{k=0}^{\infty},\left\{S_{k}\right\}_{k=0}^{\infty},\left\{a_{k}\right\}_{k=0}^{\infty}$ and $\left\{b_{k}\right\}_{k=0}^{\infty}$ be the sequences defined in $\S \S 3.2$ and let $R_{0}$ and $F_{0}$ be as in Lemma 3.3. Then we can construct $\left\{R_{k}\right\}_{k=1}^{\infty}$ and $\left\{F_{k}\right\}_{k=1}^{\infty}$ with the following properties:

(a) $R_{k-1}=R_{k}+F_{k}$ for $\tau>S_{k}$;

(b) $F_{k}$ is a solution of $L\left(S_{k}\right) F_{k}=0$ with the initial data $\tilde{R}_{k-1}\left(\in H^{1}\left(R^{3}\right)\right)$ and $\left(\widetilde{R_{k-1 \tau}}\right)$ at $\tau=S_{k}$, and

$$
F_{k}=0 \quad \text { for }|y| \leq(1-\mu)\left(\tau-S_{k}\right)-a_{k} ;
$$

(c) $R_{k}$ is a solution of problem $\left(\mathrm{Q} .1 ; S_{k}+b_{k}\right) \sim\left(\mathrm{Q} .2 ; S_{k}+b_{k}\right)$ and has compact support of at most $|y| \leq a_{k}+(1+\mu) b_{k}$ at $\tau=S_{k}+b_{k}$;

$$
\begin{aligned}
& E_{0}\left(R_{k} ; \infty, 0, S_{k}+b_{k}\right) \\
& \leq C\left(E_{0}\left(R_{k-1} ; a_{k}+(1+\mu) b_{k}, T_{k}+b_{k}-b_{k-1}, S_{k-1}+b_{k-1}\right)\right. \\
&\left.+E_{0}\left(R_{k-1} ; a_{k}+2(1+\mu) b_{k}, T_{k}-b_{k-1}, S_{k-1}+b_{k-1}\right)\right) .
\end{aligned}
$$

Proof. First we consider the case of $k=1$. Let $F_{1}$ be a solution of $L\left(S_{1}\right) F_{1}=0$ with the initial data $\tilde{R}_{0}$ and $\left(\widetilde{R_{0 \tau}}\right)$ at $\tau=S_{1}$. In other words, for $\tau>S_{1}, F_{1}$ is defined as the whole space solution of $L\left(S_{1}\right) F_{1}=0$. We continue this $F_{1}$ as $F_{1}=R_{0}$ for $\tau \leq S_{1}$. Then $F_{1}$ satisfies the equation $L(0) F_{1}=0$ in the domain exterior to $\left\{(y, \tau)|| y \mid \leq(\tau+\gamma)^{\alpha}, 0<\tau<S_{1}\right\}$. We apply Proposition 2 to $F_{1}$ in this domain. Let $\left(y_{0}, \tau_{0}\right)$ be a point with $\left|y_{0}\right| \leq(1-\mu)\left(\tau_{0}-S_{1}\right)-a_{1}, \tau_{0}>S_{1}$. According to (i) of Proposition 2 with $s=0$, the value of $F_{1}$ at $\left(y_{0}, \tau_{0}\right)$ is determined only by the value of $F_{1}$ on

$$
\left|y+a(\tau ; 0)-y_{0}-a\left(\tau_{0} ; 0\right)\right|=\tau_{0}-\tau \quad(\tau ; \text { fixed }) .
$$

Here we put $\tau=S_{0}+b_{0}=b_{0}\left(S_{0}=0\right)$. By (ii) of Proposition 2. the sphere given by the above equation with $\tau=b_{0}$ contains the sphere $\left|y-y_{0}\right|=(1-\mu)\left(\tau_{0}-b_{0}\right)$ in its interior, which, furthermore, contains the sphere $|y|=(1-\mu)\left(S_{1}-b_{0}\right)+a_{1} \quad\left(=(1-\mu)\left(T_{1}-b_{0}\right)+a_{1}, S_{1}=T_{1}\right)$. On the other hand, by Lemma 3.3, the support of $R_{0}$ at $\tau=b_{0}$ is contained in $|y| \leq a_{0}+(1+\mu) b_{0}$. In view of (3.9) in Lemma 3.2, $a_{0}+(1+\mu) b_{0}$ $<(1-\mu)\left(T_{1}-b_{0}\right)+a_{1}$ (see Fig. 1). This implies that $F_{1}=0$ for $|y|$ $\leq(1-\mu)\left(\tau-S_{1}\right)-a_{1}$, which, together with the definition of $b_{1}$ (Fig. 1), 
shows that $F_{1}$ is a solution of $\left(\mathrm{Q} .1 ; S_{1}+b_{1}\right) \sim\left(\mathrm{Q} .2 ; S_{1}+b_{1}\right)$. Thus the property (b) is established. The property (c) for $R_{1}$ follows from the fact stated above and Proposition 3.

It remains to prove (d). By property (c),

$$
\begin{aligned}
E_{0}\left(R_{1} ; \infty, 0, S_{1}+b_{1}\right)= & E_{0}\left(R_{1} ; a_{1}+(1+\mu) b_{1}, 0, S_{1}+b_{1}\right) \\
\leq & 2\left(E_{0}\left(F_{1} ; a_{1}+(1+\mu) b_{1}, 0, S_{1}+b_{1}\right)\right. \\
& \left.+E_{0}\left(R_{0} ; a_{1}+(1+\mu) b_{1}, 0, S_{1}+b_{1}\right)\right) .
\end{aligned}
$$

By definition, the second term is equal to

$$
E_{0}\left(R_{0} ; a_{1}+(1+\mu) b_{1}, T_{1}+b_{1}-b_{0}, b_{0}\right) \quad\left(S_{1}=T_{1}\right) .
$$

From Proposition 3 and the fact that $F_{1}=\tilde{R}_{0}$ and $F_{1 \tau}=\left(\widetilde{R_{0 \tau}}\right)$ at $\tau=S_{1}$, we see that

$$
E_{0}\left(F_{1} ; a_{1}+(1+\mu) b_{1}, 0, S_{1}+b_{1}\right) \leq C E_{0}\left(R_{0} ; a_{1}+2(1+\mu) b_{1}, T_{1}-b_{0}, b_{0}\right) .
$$

Thus, $F_{1}$ and $R_{1}$ with properties $(\mathrm{a}) \sim$ (d) are constructed. Repeating this procedure and noting (3.9) in Lemma 3.2, we can construct $\left\{F_{k}\right\}_{k=2}^{\infty}$ and $\left\{R_{k}\right\}_{k=2}^{\infty}$ by induction on $k$.

The following theorem is equivalent to Main Theorem stated in $\S 1$.

THEOREM 5. Suppose that Assumptions (1) $\sim(4)$ are satisfied. Let $U(y, \tau)$ be a (weak) solution of problem $(\mathrm{Q} .1 ; 0) \sim(\mathrm{Q} .2 ; 0)$ with the initial data $F(y)\left(\in H_{0}^{1}(\Omega(0))\right)$ and $G(y)\left(\in L^{2}(\Omega(0))\right)$ such that the support of $F$ and $G$ is contained in $\left\{y|y \in \Omega(0)| y \mid, \leq \gamma^{\alpha}\right\}$. Let $h>0$ be fixed and let $\mathscr{D}(\tau ; h)=\left\{y|y \in \Omega(\tau)| y \mid, \leq(\tau+\gamma)^{\alpha}+h\right\}$. Then, the local energy measured over $\mathscr{D}(\tau ; h)$ at time $\tau$ decays at the rate of $\exp \left(-M \tau^{\theta}\right)$ as $\tau \rightarrow \infty$. In other words, there exist constants $C, M$ and $\theta, 0<\theta \leq 1$, such that

$$
E_{0}\left(U ;(\tau+\gamma)^{\alpha}+h, \tau, 0\right) \leq C \exp \left(-M \tau^{\theta}\right) E_{0}(U),
$$

where $\theta=(p+1)^{-1}, p$ being the constant defined by (3.4), and

$$
E_{0}(U)=\int_{\Omega(0)}\left(|\nabla F|^{2}+G^{2}\right) d y .
$$

Proof. In virtue of (c) in Lemma 3.4, $R_{k}$ has compact support of at most $|y| \leq a_{k}+(1+\mu) b_{k}$ at $\tau=S_{k}+b_{k}$. Hence, in view of (3.6) and Lemma 3.1, there exists a constant $N$ such that

$$
a_{k}+(1+\mu) b_{k} \leq N\left(S_{k}+b_{k}+\gamma\right)^{\alpha}
$$


With this $N$, we define the constant $s_{0}=s_{0}(N, \delta)$ introduced in Theorem 1 or Theorem 4. We may assume that $S_{1}+b_{1} \geq s_{0}$ by taking $T$ (in Lemma 3.2) large enough if necessary, so that $S_{k}+b_{k} \geq s_{0}$ for $k \geq 1$.

According to Lemma 3.4, the solution $U$ may be written as

$$
U=\sum_{j=0}^{n} F_{n}+R_{n}
$$

for $\tau>S_{n}$, where

$$
F_{j}=0 \quad \text { for }|y| \leq(1-\mu)\left(\tau-S_{j}\right)-a_{j},
$$

(3.15) $R_{n}$ is a solution of problem $\left(\mathrm{Q} .1 ; S_{n}+b_{n}\right) \sim\left(\mathrm{Q} .2 ; S_{n}+b_{n}\right)$.

If $(\tau+\gamma)^{\alpha}+h<(1-\mu)\left(\tau-S_{n}\right)-a_{n}$ and $S_{n}+b_{n}<\tau$, then $F_{j}=0$ in $\mathscr{D}(\tau ; h)$, so that $U=R_{n}$ there. By this fact we have

$$
\begin{aligned}
E_{0}\left(U ;(\tau+\gamma)^{\alpha}+h, \tau, 0\right) & =E_{0}\left(R_{n} ;(\tau+\gamma)^{\alpha}+h, \tau-S_{n}-b_{n}, S_{n}+b_{n}\right) \\
& \leq E_{0}\left(R_{n} ; \infty, \tau-S_{n}-b_{n}, S_{n}+b_{n}\right) .
\end{aligned}
$$

If we note that $S_{n}+b_{n} \geq s_{0}$, then we obtain by Theorem $1^{4)}$ and Lemma 2.7 that

$$
E_{0}\left(U ;(\tau+\gamma)^{\alpha}+h, \tau, 0\right) \leq C_{0} E_{0}\left(R_{n} ; \infty, 0, S_{n}+b_{n}\right) .
$$

Furthermore, by (d) in Lemma 3.4,

$$
\begin{aligned}
& E_{0}\left(R_{n} ; \infty, 0, S_{n}+b_{n}\right) \\
& \leq \quad C\left(E_{0}\left(R_{n-1} ; a_{n}+(1+\mu) b_{n}, T_{n}+b_{n}-b_{n-1}, S_{n-1}+b_{n-1}\right)\right. \\
& \left.\quad+E_{0}\left(R_{n-1} ; a_{n}+2(1+\mu) b_{n}, T_{n}-b_{n-1}, S_{n-1}+b_{n-1}\right)\right) .
\end{aligned}
$$

Here we want to apply Theorem 4 (Remark after this theorem) to each term on the left side. To this end, we must check that $a_{n}+2(1+\mu) b_{n}$ $<\frac{1}{2}\left(T_{n}-b_{n-1}\right)$ and that the support of $R_{n-1}$ at $\tau=S_{n-1}+b_{n-1}$ is contained in $\left\{y|| y \mid \leq N\left(S_{n-1}+b_{n-1}+\gamma\right)^{\alpha}\right\}$. The first fact follows from (3.8) in Lemma 3.2 and the second one has already been established above. Hence, using (3.10) in Lemma 3.2 and the order relation for $S_{n}, a_{n}$ and $b_{n}$ (the proof of Lemma 3.2), we see that the left side of (3.16) is majorized by

$$
C_{1}\left(n^{-2 p} T^{-2}\left(n^{2 \alpha(p+1)} T^{2 \alpha}+n^{\alpha(p+1)} T^{1+\alpha}\right)+T^{\delta-\beta}\right) E_{0}\left(R_{n-1} ; \infty, 0, S_{n-1}+b_{n-1}\right) .
$$

Furthermore, recalling the definition of $p$ ((3.4) or (3.4.1), we have

4) Theorem 1 is valid for a class of weak solutions considered here. 


$$
E_{0}\left(R_{n} ; \infty, 0, S_{n}+b_{n}\right) \leq C_{2} T^{-\nu} E_{0}\left(R_{n-1} ; \infty, 0, S_{n-1}+b_{n-1}\right),
$$

where $\nu=\min (1-\alpha, \beta-\delta)>0$ and $C_{2}$ is a constant independent of $n$ and $T$. Repeating this step, we have

$$
E_{0}\left(R_{n} ; \infty, 0, S_{n}+b_{n}\right) \leq \exp \left(-(n-1) M_{1}\right) E_{0}\left(R_{1} ; \infty, 0, S_{1}+b_{1}\right),
$$

where $M_{1}=-\log \left(C_{2} T^{-\nu}\right)>0$ if $T$ is chosen large enough. And by (d) in Lemma 3.4 and by the argument used in the proof of (3.11) in Lemma 3.3 , it is not difficult to see that

$$
E_{0}\left(R_{1} ; \infty, 0, S_{1}+b_{1}\right) \leq C(T) E_{0}(U)
$$

Thus, we have

$$
E_{0}\left(U ;(\tau+\gamma)^{\alpha}+h, \tau, 0\right) \leq C(T) \exp \left(-(n-1) M_{1}\right) E_{0}(U) .
$$

Now, for given $\tau$, we choose the maximal integer $n$ such that $(\tau+\gamma)^{\alpha}+h<(1-\mu)\left(\tau-S_{n}\right)-a_{n}$ and $S_{n}+b_{n}<\tau$. Then, $n \geq C_{1}(T) \tau^{\theta}$, $\theta=(p+1)^{-1}$, for some $C_{1}(T)$. This completes the proof.

\title{
REFERENCES
}

[1] J. Cooper, Local decay of solutions of the wave equation in the exterior of a moving body, J. Math. Anal. Appl., 49 (1975), 130-153.

[2] J. Cooper and W. A. Strauss, Energy boundedness and decay of waves reflecting off a moving obstacle, India. Univ. Math. J., 25 (1976), 671-690.

[ 3 ] A. Inoue, $\operatorname{Sur} \square u+u^{3}=f$ dans un domaine non-cylindrique, J. Math. Anal. Appl., 46 (1973), 777-819.

[4] P. Lax and Phillips, Scattering Theory, Academic Press, New York, 1967.

[5] C. Morawetz, Exponential decay of solutions of the wave equation, Comm. Pure Appl. Math., 19 (1966), 439-444.

[6] H. Tamura, Local energy decays for wave equations with time-dependent coeffcients, Nagoya Math. J., 71 (1978), 107-123.

[ 7 ] E. C. Zachmanoglou, The decay of solutions of the initial-boundary value problem for the wave equation in unbounded regions, Arch. Rat. Mech. Anal., 14 (1963), $312-325$.

\author{
Department of Engineering Mathematics \\ Faculty of Engineering \\ Nagoya University
}

